\title{
X-ray Mapping and Chemical Phase Mapping with an Amptek SDD
}

\author{
L. Moran ${ }^{1}$, K. Moran ${ }^{1}$ and R. Wuhrer ${ }^{2}$ \\ ${ }^{1}$ Moran Scientific Pty Ltd, Goulburn, NSW, Australia \\ ${ }^{2}$ Advanced Materials Characterisation Facility, University of Western Sydney, NSW 2751, Australia
}

$\mathrm{X}$-ray mapping (XRM) is the collection of characteristic x-rays as a function of the position of the scanning electron beam on the specimen. This analytical technique provides a high magnification image related to the distribution and relative abundance of elements within a given specimen. Consequently, XRM has become one of the most popular and useful methods of microanalysis and has been widely implemented in many areas of research including materials science, biology, geology and environmental science.

With the development of the large area and high count rate silicon drift detectors (SDD's), Quantitative $\mathrm{X}$-ray mapping (QXRM) has become an invaluable addition to the X-ray microanalysis techniques available. The SDD's can also operate at higher beam currents, which is well suited to wavelength dispersive spectroscopy (WDS) within an electron microprobe. The larger detector area and improved count rates for electron dispersive (ED) SDD's and higher beam currents for WDS detectors considerably reduces the time to perform an x-ray map and provides the ability to capture more information and map minor and trace elements very accurately.

As such a great deal of characteristic information can be obtained from X-ray maps. Our research aims at developing post processing techniques to obtain further information about our material and make it easier for the user to obtain a better understanding of their materials unique chemical properties. Some of the post processing techniques employed include:

1. Elemental x-ray maps and quantitative X-ray maps (Fig.1a-d). Atomic number, absorption and fluorescence (ZAF) corrected quantitative X-ray maps (QXRM), where the QXRM enables reliable quantitative results, that when grouped, can be an order of magnitude better than traditional analysis [3].

2. Elemental ratio maps (Fig.1e-f). This is a powerful technique that helps to reveal subtle chemical variations between elements in a sample. Ratio maps can also be created from different combinations of elements and from the different background regions near elements of the spectra.

3. Pseudo colouring for observing element rich areas and locating phases. The colours of the elements can be rotated, which can often reveal further information (and other phases) in the material.

4. Generation of scatter diagrams and rotational scatter diagrams for locating phases [4-5]. Scatter diagrams are pixel frequency versus element concentration profiles plotted against each other in two dimensions for selected elements within the sample (Fig.1g-h).

5. Chemical phase mapping (CPM). CPM involves selecting areas on the scatter diagrams and observing where the points or clusters relate on the BSE image and is an important part of the phase identification process. These selected analysis points may then be summed for a more accurate analysis in total or by selecting strategic areas on the image.

6. Image filtering to make phase selection easier, to produce cleaner images and aid in trace element location when processing the data sets. 
Much more information about a material can be obtained by further processing the x-ray maps through data manipulation. The collection of full spectrum x-ray maps (FSXRM) and the subsequent post processing of the data to obtain quantitative X-ray maps, elemental ratio maps, scatter diagrams, pseudocoloured maps, theoretical BSE maps, totals maps as well as ZAF maps allows the user to 1) determine more information about their material through identifying the location of individual elements and phases, 2) map the spatial distribution of specific elements and chemical phases within a sample, 3) predict and verify where there is likely to be problems in the maps (images) and 4) help look at possible interface artifacts.

\section{References:}

[1] K. Moran and R. Wuhrer, Mikrochimica Acta (2006).

[2] R. Wuhrer, K. Moran, L. Moran, Materials Forum 30 (2006).

[3] Moran, K. and Wuhrer, R., Mikrochimica Acta 155 (2006), p. 59.

[4] Moran, K. and Wuhrer, R., Mikrochimica 155 (2006), p. 209.

[5] Wuhrer, R., Moran, K. and Phillips, M. R., Microscopy and Microanalysis 14 (suppl 2) (2008), $1108 \mathrm{CD}$.

[6] The authors would like to thank the Advanced Materials Characterisation Facility at the University of Western Sydney and Paul Huggett from Materials Solutions.

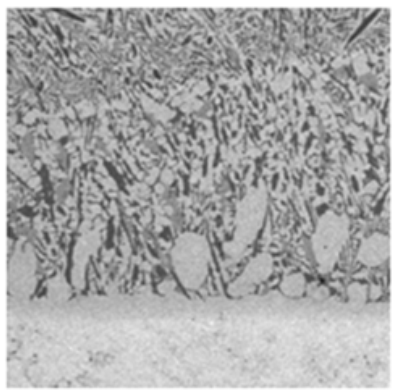

a.

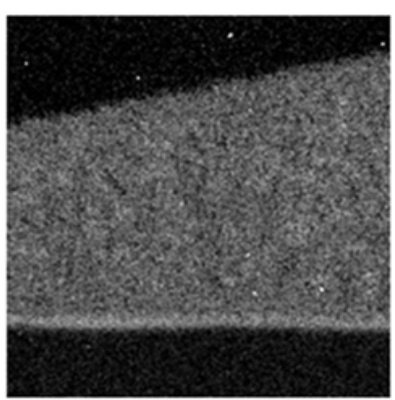

b.

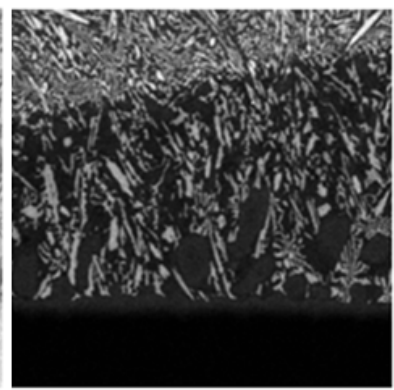

c.

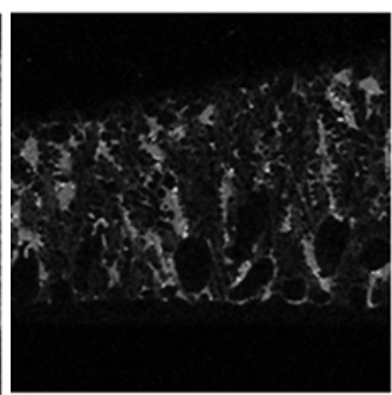

d.

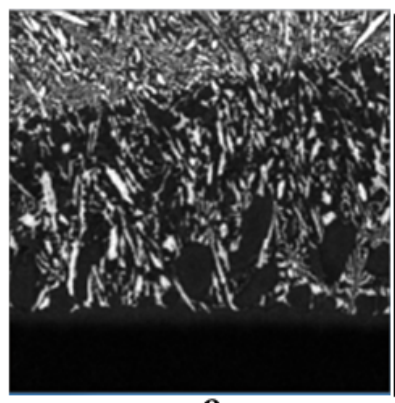

e.

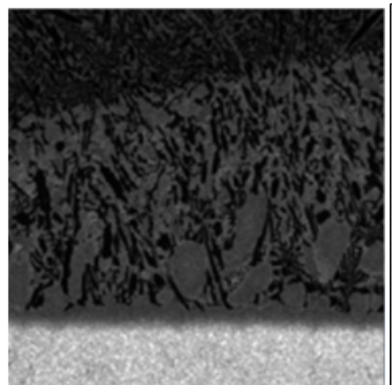

f.
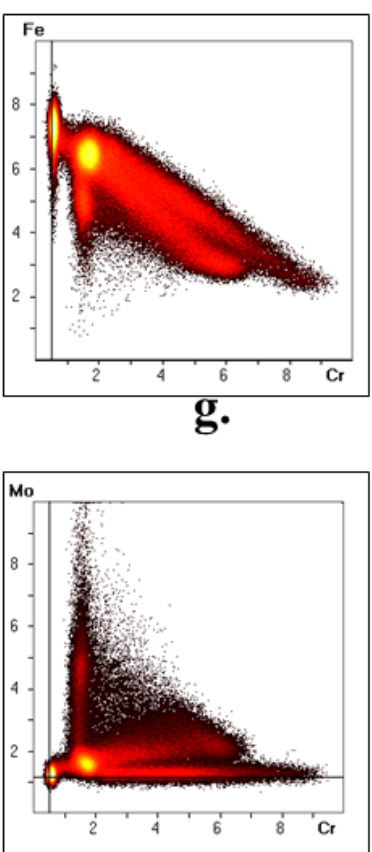

h.

Figure 1. Wide gap brazed bimetallic sample. Quantitative x-ray map of a) Iron b) Manganese, c) Chromium, d) Molybdenum, e) $\mathrm{Cr} / \mathrm{Fe}$ ratio map, f) $\mathrm{Fe} / \mathrm{Cr}$ ratio map, g) $\mathrm{Fe}-\mathrm{Cr}$ scatter diagram and h) Mo-Cr scatter diagram. Map collected at $20 \mathrm{kV}$ on a JEOL 840 SEM with a Moran microanalysis system. 